Electronic Supplementary Information (ESI)

for

\title{
Laser-Induced Optoacoustic Spectroscopy Studies of Inorganic Functionalized Metallic Nanorods
}

Paulina Błaszkiewicz ${ }^{\dagger}$, Michal Kotkowiak ${ }^{*}{ }^{\dagger}$, Emerson Coy*, Alina Dudkowiak ${ }^{\dagger}$

${ }^{\dagger}$ Faculty of Technical Physics, Poznan University of Technology, Piotrowo 3, 60-965 Poznan, Poland

NanoBioMedical Centre, Adam Mickiewicz University, Wszechnicy Piastowskiej 3, 61-614 Poznan, Poland

To whom correspondence should be addressed:

*e-mail: michal.kotkowiak@ put.poznan.pl, Tel.: +48 61665 3177, Fax: +48 616653178. 


\subsection{Chemicals}

Tetrachloroauric acid $\left(\mathrm{HAuCl}_{4} \cdot \mathrm{H}_{2} \mathrm{O}\right)(99.99 \%)$ from Alfa Aesar, cetyltrimethylammonium bromide (CTAB) $(99.00 \%)$, sodium borohydride $\left(\mathrm{NaBH}_{4}\right)(98.00 \%)$, silver nitrate $\left(\mathrm{AgNO}_{3}\right)$ (99.99\%), ascorbic acid (AA) (99.00\%), sodium hydroxide (NaOH) (99.99\%), tetraethylorthosilicate (TEOS) $(99.99 \%)$ were from Sigma Aldrich (USA). Ethanol (EtOH) $99.80 \% \mathrm{H}_{2} \mathrm{O}$ free was purchased from POCH S.A. (Poland).

\subsection{Chemical synthesis of gold nanorods and silica coating of different shell thicknesses}

All chemicals were dissolved in Mili-Q ultra-pure water in glass flasks treated with aqua regia prior to use. The synthesis of gold nanorods (Au-NRs) was performed by in situ bottomup methods, which involves the reduction of chemical precursor by a reducing agent. Synthesis of Au-NRs was a two-step process as shown in Scheme S1. The Au-NRs were synthesized by the seed-mediated growth methods, in which the grains of colloidal gold are connected to the previously obtained spherical gold nanostructures, and then around the nanostructures Au-NRs are formed. The synthesis of Au-NRs based on that proposed by Nikoobakth et al. ${ }^{1}$, was optimized and appropriately modified to meet the conditions of our lab. The first step gives a solution of spherical gold seeds (seed solution). By dissolving CTAB surfactant in deionized water we obtained a transparent solution, and then we added the precursor $\left(\mathrm{HAuCl}_{4}\right)$ and cooled reducing agent $\left(\mathrm{NaBH}_{4}\right)$. Upon vigorous stirring the colour of the solution changed to brownish-yellow. The reaction was performed in sterile conditions, while providing a constant temperature and humidity. The seed solution was stored at a temperature of approx. $28^{\circ} \mathrm{C}$, which prevented the crystallization of CTAB surfactant. The second step of the synthesis was growth of the gold seed solution (growth of Au-NRs solution). Gold seeds were added to a growth solution containing CTAB, $\mathrm{HAuCl}_{4} \times \mathrm{xH}_{2} \mathrm{O}, \mathrm{AgNO}_{3}$ and ascorbic acid. The seed solution should be added at a temperature of $27-30^{\circ} \mathrm{C}$ so that the growth of Au-NRs could take place.

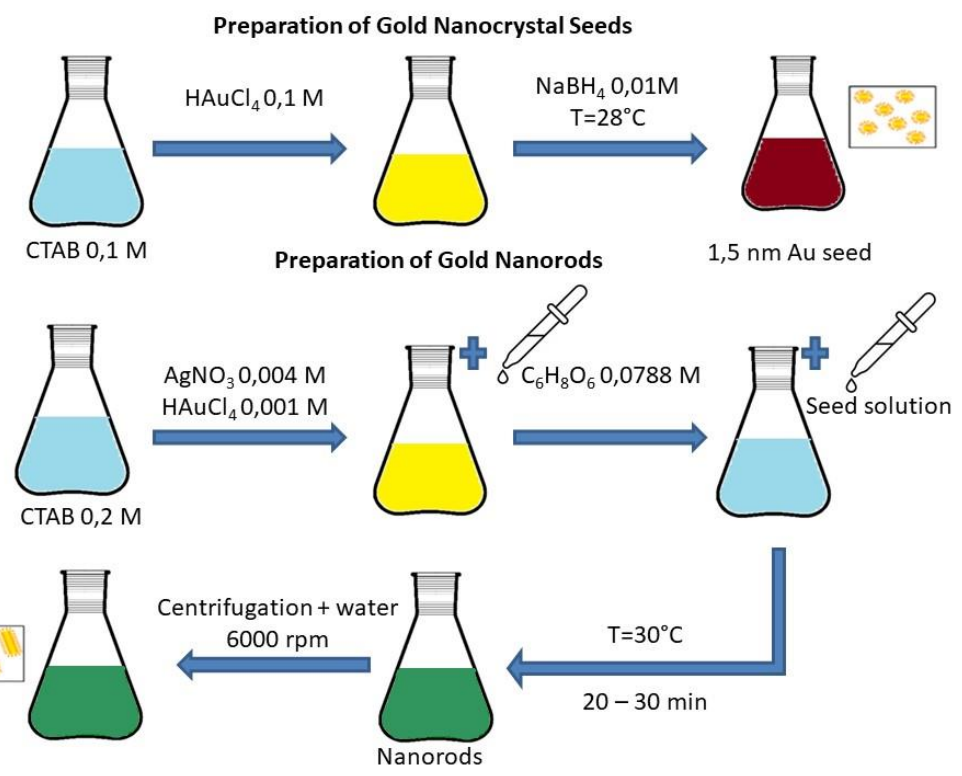

Scheme S1. Process of gold Au-NRs formation. 
The CTAB bilayer on the surface of Au-NRs inhibits their agglomerations, and the presence of $\mathrm{AgNO}_{3}$ in the growth solution encourages anisotropic Au-NRs growth. The Au-NRs with $2.25 \pm 0.23$ aspect ratio and maximum longitudinal plasmon resonance localized in the vicinity of $660 \mathrm{~nm}$ were studied. The TEM image of Au-NRs without $\mathrm{SiO}_{2}$ coating is shown in Fig. $\mathrm{S} 2$. Au-NRs for functionalization were centrifuged in order to wash excess surfactant. $\mathrm{SiO}_{2}$ coating was produced using the modified Stöber method. ${ }^{2-4}$ Concentration of CTAB surfactant must be controlled during the whole reaction process and tetraethyl orthosilicate solution (TEOS) was used for functionalization. The functionalization of Au-NRs coated with $\mathrm{SiO}_{2}$ was realized according to Scheme S2. ${ }^{2,5}$ Au-NRs were twice centrifuged at $6000 \mathrm{rpm}$ for $30 \mathrm{~min}$. After, CTAB (0.2 M) was added in different volume $(0.4,0.6,1.2$ and $1.2 \mathrm{~mL})$ and mixed 4 hours to equilibrate CTAB on the surface of Au-NRs. Then, $\mathrm{NaOH}(0.1 \mathrm{M})$ was added to the solution, that the $\mathrm{pH}$ value was obtained 10. If $\mathrm{pH}$ is below 9 then silica shell does not forms and if $\mathrm{pH}$ is higher than 10.5 it is heterogenous (Fig. S3). After 1 hour TEOS was added dropwise a 3 times in volume of $30 \mu$. TEOS was used in specific concentrations (20\% in EtOH) to obtain $\mathrm{SiO}_{2}$ shells of appropriate thicknesses. Solution was mixed overnight at $27-30^{\circ} \mathrm{C}$ and finally centrifugation twice times at $600 \mathrm{rpm}$ for $30 \mathrm{~min}$. and transferred to EtOH. In addition, $\mathrm{SiO}_{2}$ coating reduces Au-NRs aggregation, increases solubility of Au-NRs in organic solvents, and makes functionalization by various silanes an easy task.

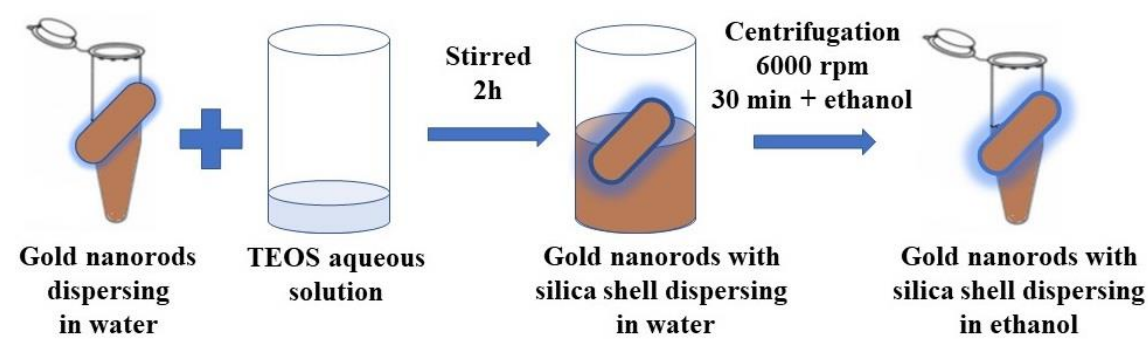

Scheme S2. Functionalization of Au-NRs with $\mathrm{SiO}_{2}$.

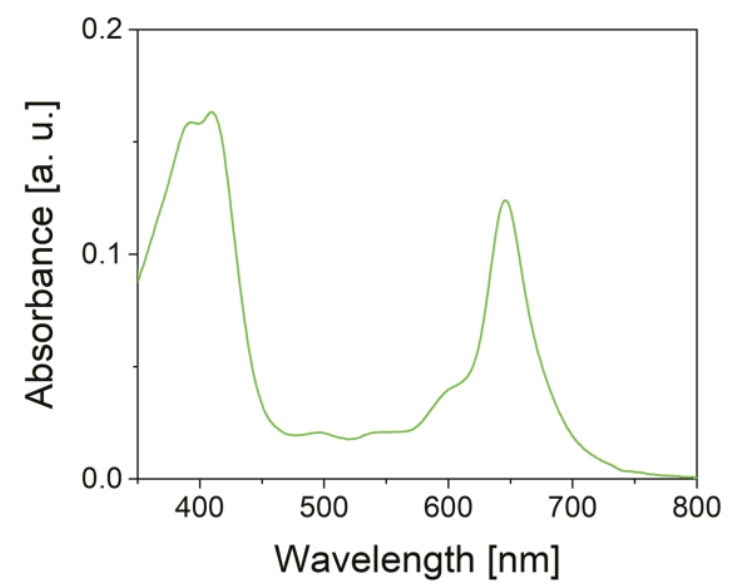

Fig. S1. UV-vis absorption spectrum of Ni-Phea in ethanol. 


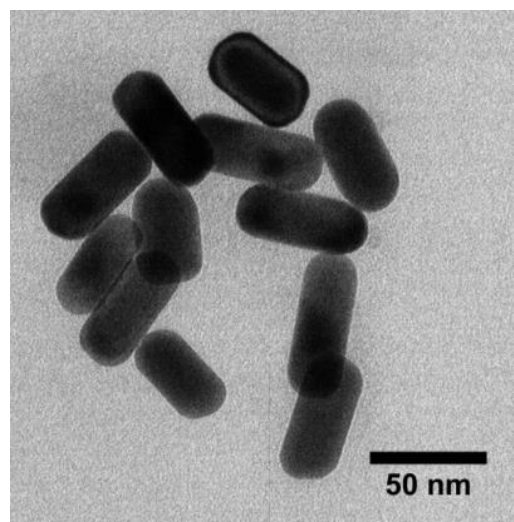

Fig. S2. TEM image of Au-NRs without $\mathrm{SiO}_{2}$ coating.

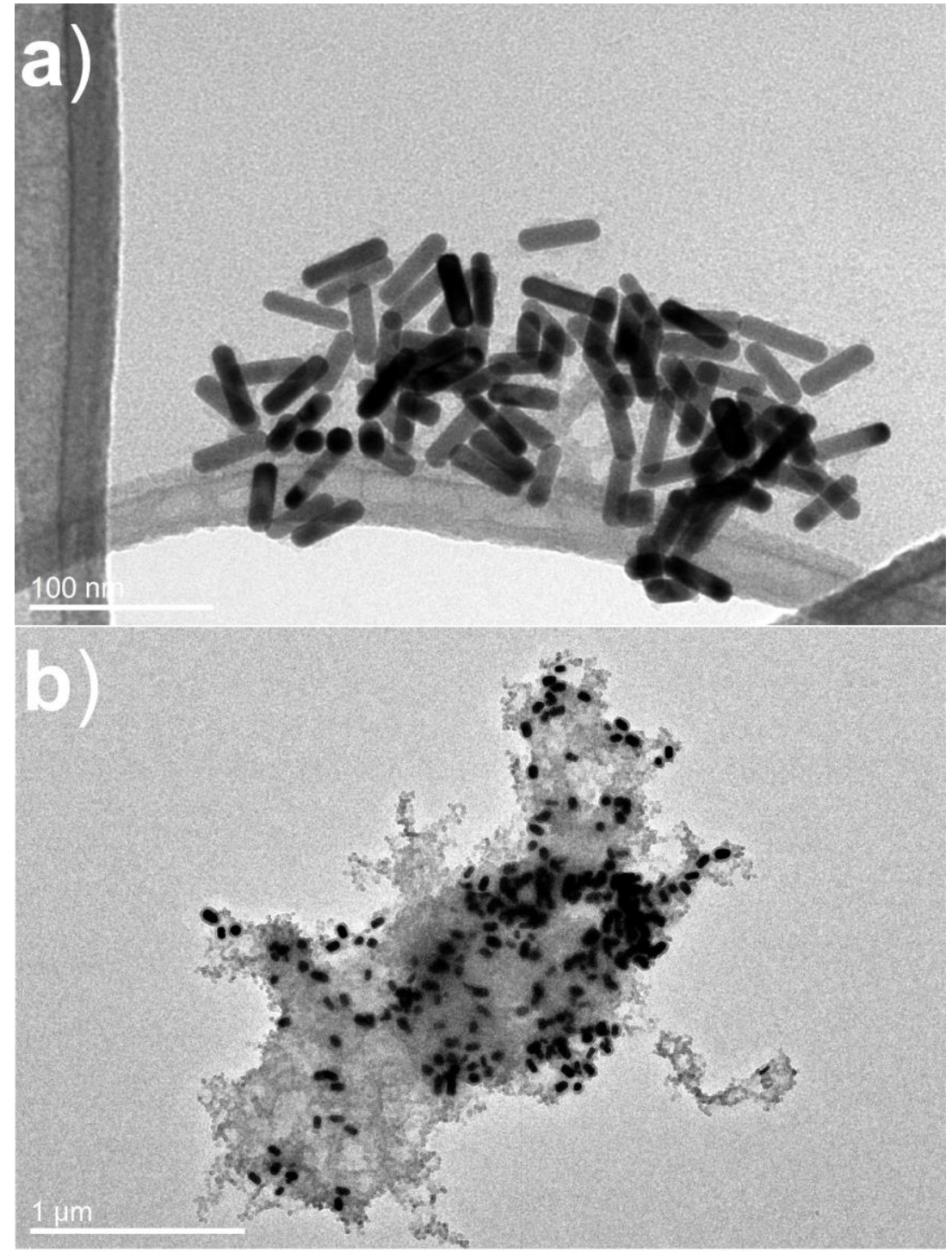

Fig. S3. TEM images of coated Au-NRs for $\mathrm{pH}$ below 9 (a) and at higher $\mathrm{pH}$ above 10.5 (b).

\section{References}

(1) Nikoobakth, B.; Sayed, A. El. Preparation and Growth Mechanism of Gold Nanorods (NRs) Using Seed-Mediated Growth Method. Chem. Mater. 2003, 15 (10), 1957-1962.

(2) Gorelikov, I.; Matsuura, N. Single-Step Coating of Mesoporous Silica on Cetyltrimethyl Ammonium Bromide-Capped Nanoparticles. Nano Lett. 2008, 8 (1), 
369-373.

(3) $\mathrm{Hu}, \mathrm{X}$; Gao, X. Multilayer Coating of Gold Nanorods for Combined Stability and Biocompatibility. Phys. Chem. Chem. Phys. 2011, 13 (21), 10028-10035.

(4) Liz-Marzán, L. M.; Giersig, M.; Mulvaney, P. Synthesis of Nanosized Gold-Silica Core-Shell Particles. Langmuir 1996, 12 (18), 4329-4335.

(5) Abadeer, N. S.; Brennan, M. R.; Wilson, W. L.; Murphy, C. J. Distance and Plasmon Wavelength Dependent Fluorescence of Molecules Bound to Silica-Coated Gold Nanorods. ACS Nano 2014, 8 (8), 8392-8406. 